\title{
BINARY SIMPLE HOMOGENEOUS STRUCTURES ARE SUPERSIMPLE WITH FINITE RANK
}

\author{
VERA KOPONEN \\ (Communicated by Mirna Džamonja)
}

\begin{abstract}
Suppose that $\mathcal{M}$ is an infinite structure with finite relational vocabulary such that every relation symbol has arity at most 2 . If $\mathcal{M}$ is simple and homogeneous, then its complete theory is supersimple with finite SU-rank which cannot exceed the number of complete 2-types over the empty set.
\end{abstract}

\section{INTRODUCTION}

A first-order structure $\mathcal{M}$ will be called homogeneous (sometimes called finitely homogeneous or ultrahomogeneous) if it is countable, has a finite vocabulary (signature) with only relation symbols (a relational vocabulary) and every isomorphism between finite substructures of $\mathcal{M}$ can be extended to an automorphism of $\mathcal{M}$. Although being countable and having a finite relational vocabulary is part of being homogeneous according to this definition, we will sometimes repeat these assumptions. If the vocabulary of $\mathcal{M}$ has only relations symbols that are unary or binary, then say that the vocabulary is binary and call $\mathcal{M}$ a binary structure. For countable $\mathcal{M}$ with finite relational vocabulary, $\mathcal{M}$ is homogeneous if and only if $\mathcal{M}$ has elimination of quantifiers [15. Corollary 7.4.2]. Via quantifier elimination one can see that infinite homogeneous structures are $\omega$-categorical [15]. Moreover, a structure is homogeneous if and only if it is the so-called Fraïssé limit of an "amalgamation class" of finite structures [10,15]. Besides being interesting objects from a model theoretic point of view, homogeneous structures have been studied in connection to Ramsey theory, constraint satisfaction problems, permutation groups and topological dynamics. See [3, 5, 14, 27, 28, for surveys of homogeneous structures and their applications.

We are far from a good understanding of homogeneous structures in general, although some particular classes of homogeneous structures have been classified or are very well understood $[5,11,12,16,20,22,23,25,31,32$. The framework of model theoretic stability theory, later generalized to simplicity theory, gives tools which makes it possible to understand structures in a quite general context. We say that an infinite structure is stable/simple if its complete first-order theory is stable/simple. Lachlan and his collaborators used tools available for stable structures to work out a very detailed understanding of infinite stable homogeneous structures;

Received by the editors June 30, 2014 and, in revised form, March 6, 2015 and April 7, 2015. 2010 Mathematics Subject Classification. Primary 03C10, 03C45, 03C50, 03C52, 03 C68.

Key words and phrases. Model theory, homogeneous structure, simple theory, stable theory, rank. 
see for example the survey 22, 11 When saying that a structure is stable or simple we will from now on assume that it is infinite. The present work and 1, 2, 19] can be seen as a continuation and (to the extent possible) generalization of the work on stable homogeneous structures. This seems worthwhile since, on the one hand, stability/simplicity theoretic ideas appear to be useful beyond the context of stable homogeneous structures, and, on the other hand, because new phenomena arise in unstable simple homogeneous structures, and these new phenomena show that the class of simple homogeneous structures is, in interesting ways, richer than the class of stable homogeneous structures. This may be of interest to applications of homogeneous structures. Some of these differences are discussed below.

All stable homogeneous structures are in fact $\omega$-stable and hence superstable. This fact follows fairly quickly from the characterizations of these notions by counting types and the fact that homogeneous structures have elimination of quantifiers. Somewhat more precisely, if $\mathcal{M}$ is (infinite) homogeneous and not $\omega$-stable, then, for some countable set $A$ the set of complete 1-types over $A$ is uncountable, and by elimination of quantifiers with respect to a finite relational vocabulary there must be an atomic formula $\varphi$ such that there are uncountably many pairwise inconsistent 1-types over $A$ which use only the formula $\varphi$. Shelah's "unstable formula theorem" [33. Ch. II, Theorem 2.2] now implies that $\mathcal{M}$ is not stable. This argument cannot be generalized to prove that every (binary) simple homogeneous structure is supersimple, because, by a well-known characterization of stability, every unstable (first-order) theory has $2^{\lambda}$ complete types over some set of parameters of cardinality $\lambda$, for every choice of infinite cardinal $\lambda$.

The following is essential for the theory of stable homogeneous structures, where $r k$ is the rank used by Lachlan which is derived from Shelah's "CP $(, 2)$-rank": if $\mathcal{M}$ is stable and homogeneous, then (a) $r k(\mathcal{M})$ is finite, and (b) every set with U-rank 1 and without a nontrivial definable equivalence relation is indiscernible 2 Neither (a) nor (b) is true in general for (even binary) simple homogeneous structures. For example, if $\mathcal{M}$ is the Rado graph, in model theory often called the random graph, then its universe is a set with U-rank 1 and without a nontrivial definable equivalence relation, but it is not indiscernible. This failure can at least partially be blamed on the failure of simple unstable structures to have "unique nondividing extensions of stationary types"; a property which all stable structures have [33, Ch. III, Corollary 2.9]. The failure of (a) for unstable infinite structures is tightly connected to Shelah's "unstable formula theorem"; see Theorem 2.2, Theorem 3.2, Definition 3.4 and Exercise 3.8 in Chapter II of [33].

In spite of the failures of (a) and (b) for unstable simple homogeneous structures, these statements seem to point in the right direction. For example, Theorem 5.1 in 2 may be seen as a version of (b) in the case of binary simple homogeneous structures. It also makes sense to use some notion of rank when studying simple homogeneous structures, as will be further discussed below. The so-called U-rank is important in many studies of stable infinite structures and since for all $\omega$-categorical

\footnotetext{
${ }^{1}$ They considered every finite homogeneous structure to be stable so also finite homogeneous structures were part of their analysis, but for the finite ones the theory is not as conclusive as for the infinite ones.

${ }^{2}$ The connection with Lachlan's terminology is the following: if the structure under consideration is stable and homogeneous, then any set which has U-rank 1 and no nontrivial definable equivalence relation is strictly minimal (and hence indiscernible).
} 
superstable $\mathcal{M}$ we have $\mathrm{U}(\mathcal{M}) \leq r k(\mathcal{M})^{3}$ it follows from (a) that every infinite stable homogeneous structure $\mathcal{M}$ has finite U-rank. The U-rank also makes sense for simple structures, but in the context of simple structures it is usually called SUrank, a convention which we follow here. If $T$ is a simple theory, then the SU-rank of a complete type (over any set of parameters) is an ordinal or $\infty$, where $\infty$ is understood to be larger than every ordinal [4,34. If every type has ordinal valued SU-rank, then the theory is called supersimple. The SU-rank of a supersimple theory $T$ is the supremum of the SU-ranks of all 1-types over $\emptyset$ (that are realized by "real elements"). Experience has shown that properties of a simple theory with finite SU-rank can often be analyzed via properties of types of SU-rank 1. For example, an $\omega$-categorical simple theory $T$ with finite SU-rank is 1-based if and only if all types of SU-rank 1 are 1-based (sometimes called modular) 13, Corollary 4.7].

All known simple homogeneous structures have complete theories which are supersimple and have finite SU-rank (and are 1-based). The most famous example is probably the random graph, which has SU-rank 1. Aranda López 1, Theorem 3.2.7] has proved that if $\mathcal{M}$ is binary, homogeneous and supersimple, then the SUrank of the complete theory of $\mathcal{M}$ cannot be $\omega^{\alpha}$ for any ordinal $\alpha \geq 1$. We are not aware of other results in this direction for simple (unstable) homogeneous structures. The main result of this article is the following, where $S_{2}(T)$ is the set of complete 2 -types over $\emptyset$ with respect to the theory $T$ :

Theorem 1. Suppose that $\mathcal{M}$ is a countable, binary, homogeneous and simple structure. Let $T$ be the complete theory of $\mathcal{M}$. Then $T$ is supersimple with finite $S U$-rank which is at most $\left|S_{2}(T)\right|$.

Since homogeneous structures have elimination of quantifiers it follows from Theorem 1 that, for every binary finite relational vocabulary $V$, every simple homogeneous $V$-structure is supersimple with SU-rank at most $2^{c|V|}$, where $c$ is a constant that depends only on $V$.

With the above theorem at hand some questions about a binary homogeneous and simple structure $\mathcal{M}$ can be studied by asking the analogous questions for types of SU-rank 1. Let $T$ be the complete theory of $\mathcal{M}$. By a result of Hart, Kim and Pillay [13, $T$ is 1-based (called 'modular' in [13]) if and only if every type of SU-rank 1 is 1-based. Moreover, by also involving work of Macpherson [26] and de Piro and Kim [9] it follows that $T$ is 1-based if and only if $T$ has trivial dependence if and only if every type of SU-rank 1 has trivial pregeometry (see for example [19, Section 2.3] for definitions and more explanation). If for every homogeneous simple structure $\mathcal{M}$ its complete theory has trivial dependence and finite SU-rank, then the behavior of dependence in simple homogeneous structures parallels that of stable homogeneous structures (see 22] for a survey of stable homogeneous structures). The reader is referred to [1,2,19] for more results about simple homogeneous structures.

It is natural to ask whether the 'binarity' assumption in Theorem 1 is necessary, especially as Cherlin and Lachlan proved that $r k(\mathcal{M})$, and hence $\mathrm{U}(\mathcal{M})$, is finite for every stable homogeneous $\mathcal{M}$ [7. Their proof relies heavily on the classification

\footnotetext{
${ }^{3}$ This inequality can be understood as follows. By [30, Corollary 6.48], U-rank coincides with Morley rank [30 Definition 6.16] if the structure is $\omega$-categorical and superstable. Moreover, it is straightforward to see that $r k(\mathcal{M})$ is at least as large as the Morley rank of $\mathcal{M}$ (where the latter is the supremum of the Morley ranks of all 1-types consistent with the complete theory of $\mathcal{M})$. Since every stable homogeneous structure $\mathcal{M}$ is $\omega$-categorical and superstable, we get $\mathrm{U}(\mathcal{M}) \leq r k(\mathcal{M})$.
} 
of finite simple groups and on the possibility, in their context, to reduce certain problems to questions about the automorphism group of a finite structure. (However, in the binary case this classification is not needed 24.) Moreover, the rank $r k$ that they consider makes sense for finite and infinite structures while the definition of SU-rank (and U-rank) presupposes that the structure in question is infinite. In addition, the reduction to finite structures in 7 may depend on a property called smooth approximability (by finite substructures) [6, 17] which holds for all stable homogeneous structures, but not for all simple homogeneous structures (the random bipartite graph is not smoothly approximable [17, p 457]). So there are some seemingly difficult obstacles if one tries to generalize Theorem 1 to nonbinary structures via the approach of [7]. On the other hand, the more advanced state of stability/simplicity theory at present compared to the 1980s may offer tools with which one can bypass or mitigate these obstacles. The proofs of this article and of [1,2,19] show that simplicity (of structures) and binarity have strong consequences when combined (via application of the "independence theorem" of simple structures [34, Theorem 2.5.20]); these consequences do not in an obvious way transfer to the context of nonbinary structures 4 But nevertheless we tend to believe that the assumption of binary vocabulary in Theorem 1 is not necessary.

The structure of this article is as follows. The next section recalls the necessary background and explains some notation and terminology. Theorem 1 is proved in Section 4. In Section 3 a technical result about independence in simple homogeneous structures is proved, which is then used in the proof of Theorem 1 .

\section{Preliminaries}

The notation and terminology that we use is more or less standard, but nevertheless we explain some notational issues here. Structures are denoted by $\mathcal{M}$ or $\mathcal{N}$ (or $\mathcal{M}^{\text {eq }}$ or $\mathcal{N}^{\text {eq }}$ if we deal with imaginaries) and their universes are denoted by $M$ or $N$ (or $M^{\text {eq }}$ or $N^{\text {eq }}$ ). Sometimes we attach indices to the letters. Finite sequences (tuples) of elements are denoted $\bar{a}, \bar{b}, \ldots$ (and finite sequences of variables $\bar{x}, \bar{y}, \ldots$ ) while $a, b, \ldots$ denote elements from some structure. By ' $\bar{a} \in A$ ' we mean that all elements in the sequence $\bar{a}$ belong to the set $A$. If we want to show that the length of $\bar{a}$ is $n$, then we may write $\bar{a} \in A^{n}$. For a set $A,|A|$ is its cardinality and for a sequence $\bar{a},|\bar{a}|$ is its length. For a sequence $\bar{a}, \operatorname{rng}(\bar{a})$ denotes the set of elements occuring in the sequence. The maximal arity of a finite relational vocabulary is, of course, the maximum of the arities of the relation symbols in the vocabulary.

Suppose that $\mathcal{M}$ is a structure, $\bar{a} \in M$ and $A \subseteq M$. Then $\operatorname{acl}_{\mathcal{M}}(A), \operatorname{dcl}_{\mathcal{M}}(A)$ and $\operatorname{tp}_{\mathcal{M}}(\bar{a} / A)$ denote the algebraic closure of $A$ with respect to $\mathcal{M}$, the definable closure of $A$ with respect to $\mathcal{M}$ and the complete type of $\bar{a}$ over $A$ with respect to $\mathcal{M}$, respectively. By $\operatorname{tp}_{\mathcal{M}}^{a t}(\bar{a} / A)$ we mean the restriction of $\operatorname{tp}_{\mathcal{M}}(\bar{a} / A)$ to atomic formulas. We often write $\operatorname{tp}_{\mathcal{M}}(\bar{a})$ instead of $\operatorname{tp}_{\mathcal{M}}(\bar{a} / \emptyset)$ (and similarly for ' $\operatorname{tp}_{\mathcal{M}}^{a t}$ '). With $\mathcal{M} \uparrow A$ we denote the substructure of $\mathcal{M}$ which is generated by $A$. Observe that if the vocabulary of $\mathcal{M}$ is relational, then $\operatorname{tp}_{\mathcal{M}}^{a t}\left(a_{1}, \ldots, a_{n}\right)=\operatorname{tp}_{\mathcal{M}}^{a t}\left(b_{1}, \ldots, b_{n}\right)$ is equivalent to saying that the map $a_{i} \mapsto b_{i}$ is an isomorphism from $\mathcal{M} \uparrow\left\{a_{1}, \ldots, a_{n}\right\}$ to $\mathcal{M} \uparrow\left\{b_{1}, \ldots, b_{n}\right\}$.

\footnotetext{
${ }^{4}$ At least one result in the binary case, namely Theorem 5.1 in 2, cannot be "naturally translated" to the nonbinary case, as witnessed by the "generic pyramid-free 3-hypergraph" which is simple with SU-rank 1; see [8, Section 3] for details.
} 
Still assume that $A \subseteq M$. By $S_{n}^{\mathcal{M}}(A)$ we denote the set of all complete $n$-types over $A$ which are realized in some elementary extension of $\mathcal{M}$. For a complete theory $T$ we let $S_{n}(T)$ be the set of all complete $n$-types (without parameters) of $T$. This means that if $\mathcal{M} \models T$, then $S_{n}(T)=S_{n}^{\mathcal{M}}(\emptyset)$. Suppose that $R \subseteq M^{k}$. Then we say that $R$ is $A$-definable (in $\mathcal{M}$ ) if there are a formula $\varphi(\bar{x}, \bar{y})$ without parameters and $\bar{a} \in A$ such that $R=\left\{\bar{b} \in M^{k}: \mathcal{M} \models \varphi(\bar{b}, \bar{a})\right\}$. A structure $\mathcal{M}$ is called $\omega$-categorical, simple or supersimple, respectively, if its complete theory, denoted $T h(\mathcal{M})$, has that property.

We refer to [15] (for example) for unexplained basic notions and notation of model theory, and to [4,34 for basic concepts and results from simplicity theory.

We have to work a little bit with imaginary elements, in order to show that we can avoid them in the crucial part of the proof of Theorem 11. As usual $\mathcal{M}^{\text {eq }}$ denotes the extension of $\mathcal{M}$ by imaginary elements. The approach to imaginary elements that we adopt is that of [15, 33] in which we do not introduce variables of different sorts but instead use unary predicates to "point out" the different sorts. This approach is also used in [2] where it is explained in more detail. The following fact which we will use is also explained in some more detail in [2].

Fact 2. Suppose that $\mathcal{M}$ is $\omega$-categorical. Then:

(i) For all $\bar{a}, \bar{b} \in M, \operatorname{tp}_{\mathcal{M}}(\bar{a})=\operatorname{tp}_{\mathcal{M}}(\bar{b})$ if and only if $\operatorname{tp}_{\mathcal{M}^{\mathrm{eq}}}(\bar{a})=\operatorname{tp}_{\mathcal{M}^{\mathrm{eq}}}(\bar{b})$ ( $\omega$-categoricity is not needed for this part).

(ii) If $B \subseteq M^{\mathrm{eq}}$ is finite and $\bar{a} \in M^{\mathrm{eq}}$, then $\operatorname{tp}_{\mathcal{M}^{\mathrm{eq}}}\left(\bar{a} / \operatorname{acl}_{\mathcal{M}^{\mathrm{eq}}}(B)\right)$ is isolated.

(iii) If $B \subseteq \mathcal{M}^{\text {eq }}$ is finite, $n<\omega$ and $p \in S_{n}^{\mathcal{M}^{\mathrm{eq}}}\left(\operatorname{acl}_{\mathcal{M}^{\mathrm{eq}}}(B)\right)$ is realized in $\mathcal{N}^{\text {eq }}$ for some $\mathcal{N} \succcurlyeq \overline{\mathcal{M}}$, then $p$ is realized in $\mathcal{M}^{\text {eq }}$.

Let $T$ be a simple theory. For every $\mathcal{M} \models T, A \subseteq M^{\text {eq }}$ and $p \in S_{n}^{\mathcal{M}^{\mathrm{eq}}}(A)$, there is a notion of SU-rank of $p$, denoted $\mathrm{SU}(p)$ (a definition is found in [4, 34]). We abbreviate $\mathrm{SU}\left(\operatorname{tp}_{\mathcal{M}^{\text {eq }}}(\bar{a} / A)\right)$ with $\mathrm{SU}(\bar{a} / A)$. For every type $p, \mathrm{SU}(p)$ is either ordinal valued or undefined (or alternatively given the value $\infty$ ). $T$ is supersimple if and only if for every $0<n<\omega$ and $p \in S_{n}(T), \mathrm{SU}(p)$ is ordinal valued (by [4, Proposition 13.13] or [34, Theorem 5.1.5], and the facts that $p \subseteq q$ implies that $\mathrm{SU}(p) \geq \mathrm{SU}(q)$ and if $\mathcal{M} \models T$ and $\bar{a} \in M^{\mathrm{eq}}$, then there is $\bar{a}^{\prime} \in M$ such that $\bar{a} \in \operatorname{dcl}_{\mathcal{M} \text { eq }}\left(\bar{a}^{\prime}\right)$ and hence $\left.\mathrm{SU}(\bar{a}) \leq \mathrm{SU}\left(\bar{a}^{\prime}\right)\right)$.

The SU-rank of $T$ is the supremum of $\left\{\mathrm{SU}(p): p \in S_{1}(T)\right\}$. If the SU-rank of $T$ is finite, then it follows from the Lascar inequalities [4,34] that $\mathrm{SU}(p)$ is finite for every $p \in S_{n}(T)$ and every $n<\omega$; so, in particular, $T$ is supersimple.

\section{An AuXiliary RESUlt about indePENDENCE}

In this section we prove a result (generalizing [2, Theorem 3.3] and its proof) which will be used in the proof of the main theorem. Actually we will only use its corollary to binary structures, but nevertheless prove the more general version since it may be useful in the future. A slightly weaker version of Corollary 6 has been proved earlier by Aranda López [1].

We consider a generalization of the independence theorem for simple theories, namely the 'strong $n$-dimensional amalgamation property for Lascar strong types', studied by Kolesnikov in [18, Definition 4.3]. In the present context of homogeneous structures, as distinct from that of Kolesnikov, a 'Lascar strong type' corresponds to a 'type over an algebraically closed set'. The notation $\mathcal{P}(S)$ denotes the powerset of $S$, and we let $\mathcal{P}^{-}(S)=\mathcal{P}(S)-\{S\}$. Every $n<\omega$ is identified with the set 
$\{0, \ldots, n-1\}$, so the notation $\mathcal{P}(n)$ makes sense. For a type $p, \operatorname{dom}(p)$ denotes the set of all parameters that occur in formulas in $p$.

Definition 3. Let $T$ be an $\omega$-categorical and simple complete theory and let $n<\omega$.

(i) A set of types $\left\{p_{s}(\bar{x}) \mid s \in \mathcal{P}^{-}(n)\right\}$ (with respect to $\mathcal{M}^{\text {eq }}$ for some $\mathcal{M} \models T$ ) is called an $n$-independent system of strong types over $A$ (where $A \subseteq \mathcal{M}^{\mathrm{eq}}$ ) if it satisfies the following properties:

(a) $\operatorname{dom}\left(p_{\emptyset}\right)=A$ and $\operatorname{dom}\left(p_{s}\right)$ is algebraically closed in $\mathcal{M}^{\text {eq }}$ for every $s \in$ $\mathcal{P}^{-}(n)$.

(b) For all $s, t \in \mathcal{P}^{-}(n)$ such that $s \subseteq t, p_{t}$ is a nondividing extension of $p_{s}$.

(c) For all $s, t \in \mathcal{P}^{-}(n), \operatorname{dom}\left(p_{s}\right) \underset{\operatorname{dom}\left(p_{s \cap t}\right)}{\perp} \operatorname{dom}\left(p_{t}\right)$.

(d) For all $s, t \in \mathcal{P}^{-}(n), p_{s}$ and $p_{t}$ extend the same type over $\operatorname{acl}_{\mathcal{M}^{\mathrm{eq}}}\left(\operatorname{dom}\left(p_{s \cap t}\right)\right)$.

(ii) We say that $T$ (and any $\mathcal{N} \models T$ ) has the $n$-dimensional amalgamation property for strong types if for every $\mathcal{M}=T$ and every $n$-independent system of strong types $\left\{p_{s}(\bar{x}) \mid s \in \mathcal{P}^{-}(n)\right\}$ over some set $A \subseteq M^{\text {eq }}$, there is a type $p^{*}$ which is a nondividing extension of $p_{s}$ for each $s \in P^{-}(n)$.

Remark 4. The independence theorem (in its general setting when the sets of parameters of the given types may be infinite [4,34]) implies that every $\omega$-categorical and simple theory has the 2-dimensional amalgamation property for strong types. (This relies on the fact that since $\omega$-categorical theories have elimination of hyperimaginaries [4,34] we can replace the 'bounded closure' with 'algebraic closure'.)

Proposition 5. Suppose that $\mathcal{M}$ has a finite relational vocabulary with maximal arity $\rho$. Also assume that $\mathcal{M}$ is countable, homogeneous and simple and has the $\rho$ dimensional amalgamation property for strong types. Let $0<n<\omega, \bar{a}_{0}, \ldots, \bar{a}_{n} \in$ $M$ and suppose that for every $s \subseteq\{0, \ldots, n\}$ such that $|s| \leq \rho,\left\{\bar{a}_{i}: i \in s\right\}$ is independent over $B \subseteq M$ and that $\operatorname{rng}\left(\bar{a}_{i}\right) \cap \operatorname{rng}\left(\bar{a}_{j}\right)=\emptyset$ whenever $i<j \leq n$. Then $\left\{\bar{a}_{0}, \ldots, \bar{a}_{n}\right\}$ is independent over $B$.

Proof. Suppose that $\mathcal{M}, \rho$ and $\bar{a}_{0}, \ldots, \bar{a}_{n} \in M$ satisfy the assumptions of the proposition. Recall that $\rho \geq 2$ by our definition in Section 2 . We use induction on $n$. The base case is when $n<\rho$ and then the conclusion is evident. So suppose that $n \geq$ $\rho$. By the induction hypothesis, every proper subset of $\left\{\bar{a}_{0}, \ldots, \bar{a}_{n}\right\}$ is independent over $B$. For a contradiction suppose that $\left\{\bar{a}_{0}, \ldots, \bar{a}_{n}\right\}$ is not independent over $B$. Then for some $i \leq n, \bar{a}_{i} \underset{B}{\chi}\left\{\bar{a}_{j}: j \leq n\right.$ and $\left.j \neq i\right\}$. Without loss of generality assume that $i=n$, so

$$
\bar{a}_{n} \underset{B}{\swarrow}\left\{\bar{a}_{i}: i<n\right\}
$$

and, by the induction hypothesis,

$$
\left\{\bar{a}_{i}: i<n\right\} \text { is independent over } B \text {. }
$$

The induction hypothesis also implies that

$$
\text { for all } s \in \mathcal{P}^{-}(n), \bar{a}_{n} \underset{B}{\downarrow}\left\{\bar{a}_{i}: i \in s\right\} .
$$

For each $s \in \mathcal{P}^{-}(\rho)$, let

$$
A_{s}=\operatorname{acl}_{\mathcal{M}^{\mathrm{eq}}}\left(\left\{\bar{a}_{i}: i \in s\right\} \cup\left\{\bar{a}_{\rho}, \ldots, \bar{a}_{n-1}\right\} \cup B\right) .
$$

Claim 1. For all $s, t \in \mathcal{P}^{-}(\rho), A_{s} \underset{A_{s \cap t}}{\perp} A_{t}$. 
Proof of Claim 1. Suppose that $s, t \in \mathcal{P}^{-}(\rho)$ and $A_{s} \underset{A_{s \cap t}}{\lfloor} A_{t}$. Then $t \backslash s \neq \emptyset$. If $|t \backslash s|>1$, then there is $t^{\prime} \subset t$ such that $t^{\prime} \cap s=t \cap s,\left|t \backslash t^{\prime}\right|=1$ and $\left|t^{\prime} \backslash s\right|>0$. By transitivity of dividing, $A_{s} \underset{A_{s \cap t^{\prime}}}{\swarrow} A_{t^{\prime}}$ or $A_{s} \underset{A_{t^{\prime}}}{\chi} A_{t}$; in the latter case $A_{s \cup t^{\prime}} \underset{A_{\left(s \cup t^{\prime}\right) \cap t}}{\chi} A_{t}$ (because $\left.\left(s \cup t^{\prime}\right) \cap t=t^{\prime}\right)$. In the first case, $\left|t^{\prime} \backslash s\right|<|t \backslash s|$. In the second case, $\left|t \backslash\left(s \cup t^{\prime}\right)\right|<|t \backslash s|$. By induction on $|t \backslash s|$ we therefore find $s^{\prime}, t^{\prime} \in \mathcal{P}^{-}(\rho)$ such that $A_{s^{\prime}} \underset{A_{s^{\prime} \cap t^{\prime}}}{\searrow} A_{t^{\prime}}$ and $\left|t^{\prime} \backslash s^{\prime}\right|=1$. By monotonicity of dividing, $A_{s^{\prime}}{\underset{B}{B}}_{A_{t^{\prime}}} A_{t^{\prime}}$ where $A_{t^{\prime}} \backslash A_{s^{\prime}}$ contains exactly one tuple from $\left\{\bar{a}_{i}: i<\rho\right\}$ (because $\left|t^{\prime} \backslash s^{\prime}\right|=1$ ). Hence $\left\{\bar{a}_{i}: i \in s^{\prime} \cup t^{\prime} \cup\{\rho, \ldots, n-1\}\right\}$ is not independent over $B$, which contradicts (2) since $s^{\prime} \cup t^{\prime} \subseteq \rho \leq n$.

Now we verify that

$$
\left\{\operatorname{tp}\left(\bar{a}_{n} / A_{s}\right): s \in \mathcal{P}(\rho)^{-}\right\}
$$

is a $\rho$-independent system of strong types over $\operatorname{acl}_{\mathcal{M}}{ }^{\mathrm{eq}}\left(B \cup\left\{\bar{a}_{\rho}, \ldots, \bar{a}_{n-1}\right\}\right)$. Properties (a) and (d) follow directly from the definition of $A_{s}$ for $s \in \mathcal{P}^{-}(\rho)$; (b) follows from (3) and monotonicity of dividing; and (c) follows from Claim 1. Since $\mathcal{M}$ has the $\rho$-dimensional amalgamation property for strong types there is $p^{*}(\bar{x})$ which is a nondividing extension of $\operatorname{tp}\left(\bar{a}_{n} / A_{s}\right)$ for each $s \in \mathcal{P}^{-}(\rho)$. Without loss of generality we may assume that $\operatorname{dom}\left(p^{*}\right)=\bigcup_{s \in \mathcal{P}^{-}(\rho)} A_{s}$. Then, by Fact 2 , we find $\bar{a} \in M$ which realizes $p^{*}$. Since $p^{*}$ does not divide over $A_{\emptyset}$ it follows that

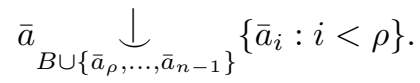

This together with (3) and transitivity gives $\bar{a} \underset{B}{\perp}\left\{\bar{a}_{i}: i<n\right\}$. To sum up, we have:

$$
\begin{aligned}
& \operatorname{tp}\left(\bar{a} / A_{s}\right)=\operatorname{tp}\left(\bar{a}_{n} / A_{s}\right) \text { for every } s \in \mathcal{P}^{-}(\rho) \text {, and } \\
& \bar{a} \bigsqcup_{B}\left\{\bar{a}_{i}: i<n\right\} .
\end{aligned}
$$

Let

$$
\begin{aligned}
C & =B \cup \operatorname{rng}\left(\bar{a}_{0}\right) \cup \ldots \cup \operatorname{rng}\left(\bar{a}_{n-1}\right) \cup \operatorname{rng}\left(\bar{a}_{n}\right) \text { and } \\
C^{\prime} & =B \cup \operatorname{rng}\left(\bar{a}_{0}\right) \cup \ldots \cup \operatorname{rng}\left(\bar{a}_{n-1}\right) \cup \operatorname{rng}(\bar{a}) .
\end{aligned}
$$

Recall that our assumptions imply that $\operatorname{rng}\left(\bar{a}_{n}\right) \cap \operatorname{rng}\left(\bar{a}_{i}\right)=\emptyset$ for all $i<n$. From (4) it follows that $\operatorname{rng}(\bar{a}) \cap \operatorname{rng}\left(\bar{a}_{i}\right)=\emptyset$ for all $i<n$.

Claim 2. The bijection $f: \mathcal{M}\left\lceil C \rightarrow \mathcal{M}\left\lceil C^{\prime}\right.\right.$ defined by $f(x)=x$ for all $x \in B \cup$ $\operatorname{rng}\left(\bar{a}_{0}\right) \cup \ldots \cup \operatorname{rng}\left(\bar{a}_{n-1}\right)$ and $f\left(\bar{a}_{n}\right)=\bar{a}$ is an isomorphism.

Proof of Claim 2. By assumption every relation symbol has arity at most $\rho$. If $\bar{b} \in M^{k}$ where $k \leq \rho$, then $\operatorname{rng}(\bar{b})$ can have nonempty intersection with at most $\rho$ of the sets $\operatorname{rng}\left(\bar{a}_{i}\right)$ for $i \leq n$, and similarly if we replace $\bar{a}_{n}$ with $\bar{a}$. Therefore (4) implies that for every relation symbol $R$ of arity $k$ and $\bar{b} \in C^{k}, \mathcal{M}\lceil C \models R(\bar{b})$ if and only if $\mathcal{M}\left\lceil C^{\prime} \models R(f(\bar{b}))\right.$, so $f$ is an isomorphism.

Since $\mathcal{M}$ is homogeneous and $B$ is finite, there is an automorphism $g$ of $\mathcal{M}$ which extends $f$ from Claim 2. Then $g\left(\bar{a}_{n}\right)=\bar{a}$ and $g$ fixes $B \cup \operatorname{rng}\left(\bar{a}_{0}\right) \cup \ldots \cup$ $\operatorname{rng}\left(\bar{a}_{n-1}\right)$ pointwise. However, since dividing is invariant under automorphisms, this contradicts (11) and the second part of (4). 
By Proposition [5 and Remark 4 we get:

Corollary 6 (A slight strengthening of [1, Proposition 3.1.4]). Suppose that $\mathcal{M}$ is binary, countable, homogeneous and simple. Let $0<n<\omega, \bar{a}_{0}, \ldots, \bar{a}_{n} \in M$, let $B \subseteq M$ be finite and suppose that for all $i<j \leq n, \bar{a}_{i} \underset{B}{\perp} \bar{a}_{j}$ and $\operatorname{rng}\left(\bar{a}_{i}\right) \cap \operatorname{rng}\left(\bar{a}_{j}\right)=$ $\emptyset$. Then $\left\{\bar{a}_{0}, \ldots, \bar{a}_{n}\right\}$ is independent over $B$.

The (anyway natural) assumption in Proposition 5 and Corollary 6 that if $i \neq j$, then $\operatorname{rng}\left(\bar{a}_{i}\right) \cap \operatorname{rng}\left(\bar{a}_{j}\right)=\emptyset$ could be removed, but at the cost of complicating the argument a little bit.

\section{Finiteness OF RANK}

In this section we prove the main result:

Theorem 1. Suppose that $\mathcal{M}$ is a countable, binary, homogeneous and simple structure. Let $T=T h(\mathcal{M})$. Then $T$ is supersimple with finite $S U$-rank which is at most $\left|S_{2}(T)\right|$.

Note that the bound on the rank need not be sharp: For every $k<\omega$ there is a binary random structure $\mathcal{M}$ such that $\left|S_{2}(T h(\mathcal{M}))\right|>k$, but the SU-rank of $T h(\mathcal{M})$ is 1. (See [2, Section 2.3] for the exact meaning of binary random structure.)

We give the proof of Theorem 1 in Section 4.1. Before that we do some preparatory work, including introducing the notion of 'preweight' which has a crucial role in the proof of Theorem 1, more precisely in Lemma 21.

Lemma 7. Suppose that $\mathcal{M}$ is a countable homogeneous and simple $V$-structure. Let $0<n<\omega$ and suppose that $A \subseteq M^{n}$ is a $\emptyset$-definable relation. Let $\mathcal{M}^{\prime}$ be the expansion of $\mathcal{M}$ to the vocabulary $V \cup\left\{R_{A}\right\}$ where $\left(R_{A}\right)^{\mathcal{M}^{\prime}}=A$. Then $\mathcal{M}^{\prime}$ is homogeneous and simple.

Proof. It is well known that simplicity is preserved if one adds relation symbols which are interpreted as relations that are $\emptyset$-definable in the original language. One way of seeing this is to consider the tree property which is equivalent to not being simple [4, 34]: If a $\left(V \cup\left\{R_{A}\right\}\right)$-formula $\varphi^{\prime}$ has the tree property with respect to $T h\left(\mathcal{M}^{\prime}\right)$, then the $V$-formula $\varphi$ obtained by replacing every occurence of $R_{A}$ with the $V$-formula which defines $A$ has the tree property with respect to $T h(\mathcal{M})$.

Now suppose that $\bar{a}, \bar{b} \in M$ and $\operatorname{tp}_{\mathcal{M}^{\prime}}^{a t}(\bar{a})=\operatorname{tp}_{\mathcal{M}^{\prime}}^{a t}(\bar{b})$. Then $\operatorname{tp}_{\mathcal{M}}^{a t}(\bar{a})=\operatorname{tp}_{\mathcal{M}}^{a t}(\bar{b})$ and as $\mathcal{M}$ is homogeneous there is an automorphism $f$ of $\mathcal{M}$ such that $f(\bar{a})=\bar{b}$. Since $A \subseteq M^{n}$ is $\emptyset$-definable, $f$ preserves $A$ setwise. Since $\left(R_{A}\right)^{\mathcal{M}^{\prime}}=A$ it follows that $f$ is an automorphism of $\mathcal{M}^{\prime}$, so $\mathcal{M}^{\prime}$ is homogeneous.

Definition 8. Suppose that $\mathcal{M}$ is a simple structure. Let $\bar{a} \in M^{\mathrm{eq}}, B \subseteq M^{\text {eq }}$ and suppose that $\kappa$ is a cardinal. The preweight of $\bar{a}$ over $B$ (with respect to $\mathcal{M}$ ), denoted $p w(\bar{a} / B)$, is at least $\kappa$ if there are $\mathcal{N} \succcurlyeq \mathcal{M}$ and a sequence $\left(\bar{a}_{i}: i<\kappa\right)$ in $\mathcal{N}^{\text {eq }}$ which is independent over $B$ and such that $\bar{a} \chi_{B} \bar{a}_{i}$ for all $i<\kappa$. We write $p w(\bar{a} / B)=\kappa$ if $p w(\bar{a} / B) \geq \kappa$ and $p w(\bar{a} / B) \nsupseteq \kappa^{+}$(where $\kappa^{+}$is the least cardinal greater than $\kappa)$.

Lemma 9 (D. Palacín [29, Lemma 2.14]). Let $V$ be a countable vocabulary. Suppose that $\mathcal{M}$ is a $V$-structure which is $\omega$-categorical and simple. Let $\bar{a} \in M^{\mathrm{eq}}$ and let $B \subseteq M^{\mathrm{eq}}$ be finite. Then $\mathrm{pw}(\bar{a} / B)<\omega$. 
4.1. Proof of Theorem 1. Let $\mathcal{M}$ be a countable, binary, simple and homogeneous structure and let $T=T h(\mathcal{M})$. Moreover, by Lemma 7 and the fact that the SU-rank of $T$ only depends on which relations in models of $T$ are $\emptyset$-definable (because of the definition of dividing), it follows that we may assume that

for every $p(x, y) \in S_{2}(T)$ there is a binary relation symbol $R_{p}$ such that $p(x, y)$ is isolated by $R_{p}(x, y)$.

Let

$$
t=\left|S_{2}(T)\right|
$$

To prove that the SU-rank of $T$ is at most $t$ we need to prove (by the finite character of dividing/forking) that there do not exist $\mathcal{N} \models T, a \in N$ and finite sets $\emptyset=$ $B_{0} \subset B_{1} \subset \ldots \subset B_{t+1} \subseteq N^{\text {eq }}$ such that $\operatorname{tp}_{\mathcal{N} \text { eq }}\left(a / B_{n+1}\right)$ divides over $B_{n}$ for every $n<t+1$. As explained at the end of Section 2 supersimplicity follows from this. The first step in the proof is to show that it suffices to consider the case when $\mathcal{N}=\mathcal{M}$ and $B_{n} \subseteq M$ for all $n \leq t+1$. This is taken care of by Lemmas 10 and 11,

Lemma 10. Suppose that there are $\mathcal{N} \models T, a \in N$ and finite subsets

$$
\emptyset=B_{0} \subset B_{1} \subset \ldots \subset B_{t+1} \subset N^{\text {eq }}
$$

such that $\operatorname{tp}_{\mathcal{N}_{\mathrm{eq}}}\left(a / B_{n+1}\right)$ divides over $B_{n}$ for every $n<t+1$. Then there are $a^{\prime} \in M$ and finite

$$
\emptyset=B_{0}^{\prime} \subset B_{1}^{\prime} \subset \ldots \subset B_{t+1}^{\prime} \subset M^{\text {eq }}
$$

such that $\operatorname{tp}_{\mathcal{M} \text { eq }}\left(a^{\prime} / B_{n+1}^{\prime}\right)$ divides over $B_{n}^{\prime}$ for every $n<t+1$.

Proof. Suppose that $\mathcal{N} \models T, a \in N$ and that $B_{n}, n \leq t+1$ satisfy the assumptions of the lemma. Without loss of generality we may assume that $\mathcal{M} \preccurlyeq \mathcal{N}$ (and $\left.\mathcal{M}^{\text {eq }} \preccurlyeq \mathcal{N}^{\text {eq }}\right)$. Let $\bar{b}_{t+1}$ enumerate $B_{t+1}$. Then $\operatorname{tp}_{\mathcal{N}^{\text {eq }}}\left(a, \bar{b}_{t+1}\right) \in S_{k}^{\mathcal{N}^{\mathrm{eq}}}(\emptyset)=S_{k}^{\mathcal{M}^{\mathrm{eq}}}(\emptyset)$ for suitably chosen $k$, so by Fact 2, $\operatorname{tp}_{\mathcal{N} \text { eq }}\left(a, \bar{b}_{t+1}\right)$ is also realized in $\mathcal{M}^{\text {eq }}$ by some $a^{\prime}, \bar{b}_{t+1}^{\prime} \in M^{\text {eq }}$. Then $\operatorname{rng}\left(\bar{b}_{t+1}^{\prime}\right)$ contains sets $B_{n}^{\prime}$, for $n \leq t+1$, which satisfy the conclusion of the lemma.

Lemma 11. Suppose that there are $a \in M$ and finite subsets

$$
\emptyset=B_{0} \subset B_{1} \subset \ldots \subset B_{t+1} \subset M^{\text {eq }}
$$

such that $\operatorname{tp}_{\mathcal{M} \text { eq }}\left(a / B_{n+1}\right)$ divides over $B_{n}$ for every $n<t+1$. Then there are finite subsets

$$
\emptyset=B_{0}^{\prime} \subset B_{1}^{\prime} \subset \ldots \subset B_{t+1}^{\prime} \subset M
$$

such that $\operatorname{tp}_{\mathcal{M}}\left(a / B_{n+1}^{\prime}\right)$ divides over $B_{n}^{\prime}$ for every $n<t+1$.

Proof. Suppose that there are $a \in M$ and finite subsets $\emptyset=B_{0} \subset B_{1} \subset \ldots \subset$ $B_{t+1} \subset M^{\text {eq }}$ such that $\operatorname{tp}_{\mathcal{M}^{\text {eq }}}\left(a / B_{n+1}\right)$ divides over $B_{n}$ for every $n<t+1$. For every $n \leq t+1$ there is finite $B_{n}^{\prime} \subset M$ such that $B_{n} \subseteq \operatorname{dcl}_{\mathcal{M}^{\mathrm{eq}}}\left(B_{n}^{\prime}\right)$. By enumerating $B_{n}^{\prime}$ as $\bar{b}_{n}^{\prime}$ and using the existence of a nondividing extension of $\operatorname{tp}_{\mathcal{M}^{\text {eq }}}\left(\bar{b}_{n}^{\prime} / B_{n}\right)$ to $B_{n} \cup\{a\}$ (and Fact 2) we may also assume that $a \underset{B_{n}}{\frac{1}{4}} B_{n}^{\prime}$ for all $n \leq t+1$. Suppose for a contradiction that $a \underset{B_{n}^{\prime}}{\perp} B_{n+1}^{\prime}$ for some $n<t+1$. Then $a \underset{B_{n} B_{n}^{\prime}}{\perp} B_{n+1}^{\prime}$ because $B_{n} \subseteq$ $\operatorname{dcl}_{\mathcal{M}^{\mathrm{eq}}}\left(B_{n}^{\prime}\right)$. By transitivity of dividing (and since $a \underset{B_{n}}{\perp} \stackrel{B_{n}^{\prime}}{\prime}$ ) we get $a \frac{\downarrow}{B_{n}} B_{n}^{\prime} B_{n+1}^{\prime}$, so $a \underset{B_{n}}{\perp} B_{n+1}^{\prime}$. As $B_{n+1} \subseteq \operatorname{dcl}_{\mathcal{M}^{\mathrm{eq}}}\left(B_{n+1}^{\prime}\right)$ we have $a \underset{B_{n}}{\perp} B_{n+1}$ which contradicts the assumption. Hence $a \underset{B_{n}^{\prime}}{\chi_{n+1}} B_{n}^{\prime}$ for every $n<t+1$. Note that the construction does 
not guarantee that $B_{n}^{\prime} \subseteq B_{n+1}^{\prime}$. But by letting $B_{n}^{\prime \prime}=\bigcup_{i \leq n} B_{n}^{\prime}$ we get $B_{n}^{\prime \prime} \subseteq B_{n+1}^{\prime \prime}$ and still have $a \underset{B_{n}^{\prime \prime}}{\backslash} B_{n+1}^{\prime \prime}$ for all $n<t+1$.

From Lemmas 10 and11 it follows that to prove that the SU-rank of $T=T h(\mathcal{M})$ is at most $t$ it suffices to prove:

Lemma 12. There do not exist $a \in M$ and finite subsets

$$
\emptyset=B_{0} \subset B_{1} \subset \ldots \subset B_{t+1} \subset M
$$

such that $\operatorname{tp}_{\mathcal{M}}\left(a / B_{n+1}\right)$ divides over $B_{n}$ for every $n<t+1$.

Towards a contradiction,

(6) assume that $a \in M$ and there are finite $\emptyset=B_{0} \subset B_{1} \subset \ldots \subset B_{t+1} \subset M$ such that $\operatorname{tp}_{\mathcal{M}}\left(a / B_{n+1}\right)$ divides over $B_{n}$ for every $n<t+1$.

We will derive a contradiction via a construction of homogeneous simple substructures $\mathcal{M}=\mathcal{M}_{0} \supset \mathcal{M}_{1} \supset \ldots \supset \mathcal{M}_{t+1}$ and an argument which is divided into a few lemmas.

Notation 13. We will consider dividing in different structures where the universe of one is included in another. To distinguish which structure we have in mind we use the following notation: if $\mathcal{N}$ is a structure, $\bar{a}, \bar{b} \in N$ and $C \subseteq N$, then $\bar{a}{\underset{C}{C}}_{\perp} \mathcal{N} \bar{b}$ means that $\bar{a}$ is independent from $\bar{b}$ over $C$ in $\mathcal{N}$ (or "with respect to $\mathcal{N}$ ").

Recall that the (finite) vocabulary of $\mathcal{M}$ is denoted $V$.

Definition 14. Let $p_{1}(x, y), \ldots, p_{t}(x, y)$ be an enumeration of $S_{2}(T)$. By assumption (5), there are binary relation symbols $R_{1}, \ldots, R_{t} \in V$ such that for each $i$, $R_{i}(x, y)$ isolates $p_{i}(x, y)$.

Note that, since $\mathcal{M}$ is homogeneous, every $p \in S_{2}(T)$ is realized in $\mathcal{M}$.

Definition 15. Let $\mathcal{N}$ be a simple $V$-structure and let $R \in V$ be binary.

We call $R$ a dividing relation with respect to $\mathcal{N}$ if for all $a, b \in N, \mathcal{N} \models R(a, b)$ implies $a \swarrow^{\mathcal{N}} b$. We call $R$ a nondividing relation with respect to $\mathcal{N}$ if for all $a, b \in N$, $\mathcal{N} \models R(a, b)$ implies $a \perp^{\mathcal{N}} b$.

Note that, in general, a binary $R \in V$ may be neither a dividing relation with respect to $\mathcal{N}$ nor a nondividing relation with respect to $\mathcal{N}$.

Definition 16. Let $\mathcal{M}_{0}=\mathcal{M}$ and $T_{0}=T$. For all $n=1, \ldots, t+1$, let

$$
\begin{aligned}
M_{n} & =\left\{a^{\prime} \in M: \operatorname{tp}_{\mathcal{M}}\left(a^{\prime} / B_{n}\right)=\operatorname{tp}_{\mathcal{M}}\left(a / B_{n}\right)\right\}, \\
\mathcal{M}_{n} & =\mathcal{M} \uparrow M_{n}, \text { and } \\
T_{n} & =\operatorname{Th}\left(\mathcal{M}_{n}\right) .
\end{aligned}
$$

Hence each $\mathcal{M}_{n}$ is a substructure of $\mathcal{M}$ and thus a $V$-structure. Also note that

$$
\mathcal{M}=\mathcal{M}_{0} \supset \mathcal{M}_{1} \supset \ldots \supset \mathcal{M}_{t+1}
$$

and that $M_{t}$ is infinite, because $\operatorname{tp}_{\mathcal{M}}\left(a / B_{t+1}\right)$ divides over $B_{t}$ and therefore $\operatorname{tp}_{\mathcal{M}}\left(a / B_{t}\right)$ cannot be algebraic.

Lemma 17. For all $n=0, \ldots, t, \mathcal{M}_{n}$ is simple and homogeneous. 
Proof. The case $n=0$ is trivial, so suppose that $1 \leq n \leq t$. If a simple structure is expanded with constant symbols (but nothing more), then the resulting expansion is also simple, by [4, Remark 2.26] for example. Every infinite structure which is interpretable in a simple structure is simple, by [34, Corollary 2.8.11] for example. By the $\omega$-categoricity of $\mathcal{M}, \operatorname{tp}_{\mathcal{M}}\left(a / B_{n}\right)$ is isolated (recall that $B_{n}$ is finite) and therefore $M_{n}$ is $B_{n}$-definable in $\mathcal{M}$. It follows that $\mathcal{M}_{n}$ is interpretable in the expansion of $\mathcal{M}$ with constants for elements in $B_{n}$. Thus $\mathcal{M}_{n}$ is simple.

For homogeneity, suppose that $\bar{a}=\left(a_{1}, \ldots, a_{k}\right), \bar{b}=\left(b_{1}, \ldots, b_{k}\right) \in\left(M_{n}\right)^{k}$ and $\operatorname{tp}_{\mathcal{M}_{n}}^{a t}(\bar{a})=\operatorname{tp}_{\mathcal{M}_{n}}^{a t}(\bar{b})$. As $\mathcal{M}_{n}$ is a substructure of $\mathcal{M}$ we get

$$
\operatorname{tp}_{\mathcal{M}}^{a t}(\bar{a})=\operatorname{tp}_{\mathcal{M}}^{a t}(\bar{b})
$$

Since $\bar{a}, \bar{b} \in\left(M_{n}\right)^{k}$ we also have

$$
\operatorname{tp}_{\mathcal{M}}\left(a_{i} / B_{n}\right)=\operatorname{tp}_{\mathcal{M}}\left(b_{i} / B_{n}\right)=\operatorname{tp}_{\mathcal{M}}\left(a / B_{n}\right) \text { for all } i=1, \ldots, k .
$$

Since $\mathcal{M}$ is binary we get $\operatorname{tp}_{\mathcal{M}}^{a t}\left(\bar{a} / B_{n}\right)=\operatorname{tp}_{\mathcal{M}}^{a t}\left(\bar{b} / B_{n}\right)$ and as $\mathcal{M}$ is homogeneous there is an automorphism $f$ of $\mathcal{M}$ such that $f(\bar{a})=\bar{b}$ and $f$ fixes $B_{n}$ pointwise. Since $M_{n}$ is $B_{n}$-definable in $\mathcal{M}$, it follows that $f$ fixes $M_{n}$ setwise. Hence $f\left\lceil M_{n}\right.$ is an automorphism of $\mathcal{M}_{n}=\mathcal{M} \uparrow M_{n}$.

Corollary 18. For all $i=1, \ldots, t$ and all $n=0,1, \ldots, t, R_{i}(x, y)$ isolates a type in $S_{2}\left(T_{n}\right)$. Moreover, every type in $S_{2}\left(T_{n}\right)$ is isolated by some $R_{i}$.

Proof. Since $\mathcal{M}$ is homogeneous and $\mathcal{M}_{n} \subseteq \mathcal{M}$ it follows from Definition 14 that for every $i=1, \ldots, t$ and every atomic $V$-formula $\varphi(x, y)$,

$$
\mathcal{M}_{n} \models \forall x, y\left(R_{i}(x, y) \rightarrow \varphi(x, y)\right) \vee \forall x, y\left(R_{i}(x, y) \rightarrow \neg \varphi(x, y)\right) .
$$

By Lemma 17, $\mathcal{M}_{n}$ is homogeneous and hence it has elimination of quantifiers, so the first claim of the corollary follows. The second claim is just a restatement of what is said in Definition 14

Corollary 18 immediately implies the following:

Corollary 19. For all $i=1, \ldots, t$ and all $n=0,1, \ldots, t, R_{i}$ is a dividing relation or a nondividing relation (but not both) with respect to $\mathcal{M}_{n}$.

Lemma 20. For all $n=0, \ldots, t$ and all $c, d \in M_{n}, c \downarrow \mathcal{M}^{n} d$ if and only if $c_{B_{n}} \mathcal{M}_{d}$.

Proof. Since $B_{0}=\emptyset$ and $\mathcal{M}_{0}=\mathcal{M}$ the lemma is trivial for $n=0$. Let $1 \leq$ $n \leq t$ and $c, d \in M_{n}$. Suppose that $c \swarrow^{\mathcal{M}_{n}} d$. By the definition of dividing there are a $V$-formula $\varphi(x, y)$ (without parameters) and $d_{i} \in M_{n}$ for $i<\omega$ such that $\varphi(x, y) \in \operatorname{tp}_{\mathcal{M}_{n}}(c, d), \operatorname{tp}_{\mathcal{M}_{n}}\left(d_{i}\right)=\operatorname{tp}_{\mathcal{M}_{n}}(d)$ for all $i$ and $\left\{\varphi\left(x, d_{i}\right): i<\omega\right\}$ is $k$ inconsistent (with respect to $T_{n}=\operatorname{Th}\left(\mathcal{M}_{n}\right)$ ) for some $k<\omega$. (It follows from the homogeneity of $\mathcal{M}_{n}$ and Fact 2 that such $d_{i}$ can be found in $\mathcal{M}_{n}$.) Since $\mathcal{M}_{n}$ is homogeneous we may assume that $\varphi$ is quantifier free. By the definition of $\mathcal{M}_{n}$ we have $\operatorname{tp}_{\mathcal{M}}\left(d_{i} / B_{n}\right)=\operatorname{tp}_{\mathcal{M}}\left(d / B_{n}\right)$ for all $i$.

Since $\mathcal{M}_{n} \subseteq \mathcal{M}, \varphi(x, y) \in \operatorname{tp}_{\mathcal{M}_{n}}(c, d)$ and $\varphi(x, y)$ is quantifier free it follows that

$$
\varphi(x, y) \in \operatorname{tp}_{\mathcal{M}}(c, d) .
$$

Let $\bar{b}_{n}$ be an enumeration of $B_{n}$ and let $\psi\left(x, y, \bar{b}_{n}\right)$ isolate $\operatorname{tp}_{\mathcal{M}}\left(c, d / B_{n}\right)$. By the homogeneity of $\mathcal{M}$ we may assume that $\psi$ is quantifier free. Then let $\varphi^{\prime}\left(x, y, \bar{b}_{n}\right)$ be the (quantifier free) formula

$$
\varphi(x, y) \wedge \psi\left(x, y, \bar{b}_{n}\right)
$$


so $\varphi^{\prime}\left(x, d, \bar{b}_{n}\right) \in \operatorname{tp}_{\mathcal{M}}\left(c /\{d\} \cup B_{n}\right)$. If $\left\{\varphi^{\prime}\left(x, d_{i}, \bar{b}_{n}\right): i<\omega\right\}$ would not be $k$ inconsistent with respect to $\mathcal{M}$, then there would be $i_{1}, \ldots, i_{k}$ and $c^{\prime} \in M$ such that

$$
\mathcal{M}=\bigwedge_{j=1}^{k} \varphi\left(c^{\prime}, d_{i_{j}}, \bar{b}_{n}\right),
$$

which, by the definitions of $\mathcal{M}_{n}$ and $\varphi^{\prime}$, implies $c^{\prime} \in M_{n}$ and $\mathcal{M}_{n} \models \bigwedge_{j=1}^{k} \varphi\left(c^{\prime}, d_{i_{j}}\right)$. Then $\left\{\varphi\left(x, d_{i}\right): i<\omega\right\}$ is not $k$-inconsistent with respect to $\mathcal{M}_{n}$, contradicting our assumptions. Hence $\left\{\varphi^{\prime}\left(x, d_{i}, \bar{b}_{n}\right): i<\omega\right\}$ is $k$-inconsistent with respect to $\mathcal{M}$ and therefore $c \underset{B_{n}}{\not} \mathcal{M} d$.

Now suppose that $c \underset{B_{n}}{\not} \mathcal{M} d$ and let $\bar{b}_{n}$ enumerate $B_{n}$. Then there are $\varphi\left(x, y, \bar{b}_{n}\right) \in$ $\operatorname{tp}_{\mathcal{M}}\left(c, d / B_{n}\right)$ and $d_{i} \in M$ for $i<\omega$ such that $\operatorname{tp}_{\mathcal{M}}\left(d_{i} / B_{n}\right)=\operatorname{tp}_{\mathcal{M}}\left(d / B_{n}\right)$ for all $i$ and $\left\{\varphi\left(x, d_{i}, \bar{b}_{n}\right): i<\omega\right\}$ is $k$-inconsistent with respect to $\mathcal{M}$ for some $k<\omega$. By homogeneity of $\mathcal{M}$ we may assume that $\varphi$ is quantifier free. Recall that we assume that $c, d \in M_{n}$. Since $\operatorname{tp}_{\mathcal{M}}\left(d_{i} / B_{n}\right)=\operatorname{tp}_{\mathcal{M}}\left(d / B_{n}\right)$ we have $d_{i} \in M_{n}$ for all $i$. Since $\mathcal{M}_{n} \subseteq \mathcal{M}$ where both structures are homogeneous we also get

$$
\operatorname{tp}_{\mathcal{M}_{n}}\left(d_{i}\right)=\operatorname{tp}_{\mathcal{M}_{n}}(d) \text { for all } i .
$$

Let $\psi(x, y)$ isolate $\operatorname{tp}_{\mathcal{M}_{n}}(c, d)$. As $\mathcal{M}_{n}$ is homogeneous we may assume that $\psi$ is quantifier free.

Suppose for a contradiction that $\left\{\psi\left(x, d_{i}\right): i<\omega\right\}$ is not $k$-inconsistent with respect to $\mathcal{M}_{n}$. Then there are $i_{1}, \ldots, i_{k}$ and $c^{\prime} \in M_{n}$ such that $\mathcal{M}_{n} \models \bigwedge_{j=1}^{k} \psi\left(c^{\prime}, d_{i_{j}}\right)$. Because $\psi$ is quantifier free and $\mathcal{M}_{n} \subseteq \mathcal{M}$ we get $\mathcal{M} \models \bigwedge_{j=1}^{k} \psi\left(c^{\prime}, d_{i_{j}}\right)$. Since $\psi$ isolates $\operatorname{tp}_{\mathcal{M}_{n}}(c, d)$ and $c^{\prime}, c, d, d_{i_{j}} \in M_{n}$ where $\mathcal{M}_{n} \subseteq \mathcal{M}$ it follows that $\operatorname{tp}_{\mathcal{M}}^{a t}\left(c^{\prime}, d_{i_{j}}\right)=$ $\operatorname{tp}_{\mathcal{M}}^{a t}(c, d)$ (for all $\left.j\right)$. By the definition of $M_{n}$ we have $\operatorname{tp}_{\mathcal{M}}\left(c^{\prime} / B_{n}\right)=\operatorname{tp}_{\mathcal{M}}\left(c / B_{n}\right)=$ $\operatorname{tp}_{\mathcal{M}}\left(d / B_{n}\right)=\operatorname{tp}_{\mathcal{M}}\left(d_{i_{j}} / B_{n}\right)$. Since $\mathcal{M}$ is binary it follows that $\operatorname{tp}_{\mathcal{M}}^{a t}\left(c^{\prime}, d_{i_{j}} / B_{n}\right)=$ $\operatorname{tp}_{\mathcal{M}}^{a t}\left(c, d / B_{n}\right)$ which by the homogeneity of $\mathcal{M}$ gives $\operatorname{tp}_{\mathcal{M}}\left(c^{\prime}, d_{i_{j}} / B_{n}\right)=\operatorname{tp}_{\mathcal{M}}\left(c, d / B_{n}\right)$ for all $j$. Consequently $\mathcal{M} \models \bigwedge_{j=1}^{k} \varphi\left(c^{\prime}, d_{i_{j}}, \bar{b}_{n}\right)$ so $\left\{\varphi\left(x, d_{i}, \bar{b}_{n}\right): i<\omega\right\}$ is not $k$ inconsistent with respect to $\mathcal{M}$, contradicting the assumption. Hence $\left\{\psi\left(x, d_{i}\right)\right.$ : $i<\omega\}$ is $k$-inconsistent with respect to $\mathcal{M}_{n}$ and therefore $c \chi^{\mathcal{M}_{n}} d$.

Lemma 21. Let $1 \leq n \leq t$. There is $1 \leq s \leq t$ such that $R_{s}$ is a nondividing relation with respect to $\mathcal{M}_{n}$ and, for every $m<n, R_{s}$ is a dividing relation with respect to $\mathcal{M}_{m}$.

Proof. Recall that for every $n=0, \ldots, t, \mathcal{M}_{n}$ is infinite and (by Lemma 17) simple and homogeneous. Fix any $1 \leq n \leq t$. Let $\bar{b}_{n}$ enumerate $B_{n}$. For every $0 \leq m<n$ let $\alpha_{m}=p w\left(\bar{b}_{n} / B_{m}\right)$ where the preweight is taken with respect to $\mathcal{M}=\mathcal{M}_{0}$. By Lemma 9, $\alpha_{m}<\omega$ for every $m<n$. Then let $\alpha=\max \left\{\alpha_{0}, \ldots, \alpha_{n-1}\right\}$, so $\alpha<\omega$.

By repeatedly applying the existence of nondividing extensions and Fact 2 to $\mathcal{M}_{n}$ it follows that there are $c_{i} \in M_{n}$ for $i<\omega$ such that $\left\{c_{i}: i<\omega\right\}$ is an independent set over $\emptyset$ with respect to $\mathcal{M}_{n}$. By Ramsey's theorem [15, Theorem 11.1.3 or its corollary] there are distinct $k_{0}<\ldots<k_{\alpha}<\omega$ such that

$$
\operatorname{tp}_{\mathcal{M}_{n}}\left(c_{k_{i}}, c_{k_{j}}\right)=\operatorname{tp}_{\mathcal{M}_{n}}\left(c_{k_{i^{\prime}}}, c_{k_{j^{\prime}}}\right)
$$

whenever $0 \leq i<j \leq \alpha$ and $0 \leq i^{\prime}<j^{\prime} \leq \alpha$. In other words, by renaming elements for notational simplicity, we have found distinct $d_{0}, \ldots, d_{\alpha} \in M_{n}$ such 
that $\left\{d_{0}, \ldots, d_{\alpha}\right\}$ is independent over $\emptyset$ with respect to $\mathcal{M}_{n}$ and $\operatorname{tp}_{\mathcal{M}_{n}}\left(d_{i}, d_{j}\right)=$ $\operatorname{tp}\left(d_{i^{\prime}}, d_{j^{\prime}}\right)$ whenever $0 \leq i<j \leq \alpha$ and $0 \leq i^{\prime}<j^{\prime} \leq \alpha$. By Corollary [18] for some $1 \leq s \leq t, R_{s}$ isolates $\operatorname{tp}_{\mathcal{M}_{n}}\left(d_{i}, d_{j}\right)$ for all $0 \leq i<j \leq \alpha$. Hence $\mathcal{M}_{n} \models R_{s}\left(d_{i}, d_{j}\right)$ and thus $\mathcal{M}_{m} \models R_{s}\left(d_{i}, d_{j}\right)$ for all $m<n$ and all $0 \leq i<j \leq \alpha$ (because $\mathcal{M}_{n} \subseteq \mathcal{M}_{m}$ if $m<n)$. As $\mathcal{M}=\mathcal{M}_{0}$ we have $\mathcal{M} \models R_{s}\left(d_{i}, d_{j}\right)$ for all $0 \leq i<j \leq \alpha$. Since $\left\{d_{0}, \ldots, d_{\alpha}\right\}$ is independent over $\emptyset$ in $\mathcal{M}_{n}$ it follows that $R_{s}$ is a nondividing relation with respect to $\mathcal{M}_{n}$.

Let $m<n$ and suppose for a contradiction that $R_{s}$ is a nondividing relation with respect to $\mathcal{M}_{m}$. Then $d_{i}\left\lfloor\mathcal{M}_{m} d_{j}\right.$ for all $0 \leq i<j \leq \alpha$, which by Lemma 20 gives $d_{i} \underset{B_{m}}{\perp} \mathcal{M} d_{j}$ for all $0 \leq i<j \leq \alpha$. Now Corollary 6 implies that $\left\{d_{0}, \ldots, d_{\alpha}\right\}$ is independent over $B_{m}$ in $\mathcal{M}$. By assumption (6), the definition of $\mathcal{M}_{n}$ and since $d_{i} \in M_{n}$ and $n>m$ (so $B_{m} \subseteq B_{n-1}$ ) we also have $d_{i} \underset{B_{m}}{\chi} \mathcal{M} \bar{b}_{n}$, for every $i \leq \alpha$. But as $\left|\left\{d_{0}, d_{1}, \ldots, d_{\alpha}\right\}\right|>\alpha \geq \alpha_{m}$, this contradicts the assumption that $p w\left(\bar{b}_{n} / B_{m}\right)=\alpha_{m}$. Hence $R_{s}$ is (by Corollary 19) a dividing relation with respect to $\mathcal{M}_{m}$.

With Lemma 21 we can now derive a contradiction which proves Lemma 12 and hence also Theorem 1. By Lemma 21, there is $1 \leq s \leq t$ such that $R_{s}$ is a nondividing relation with respect to $\mathcal{M}_{t}$ and a dividing relation with respect to $\mathcal{M}_{n}$ for every $n<t$. Without loss of generality (by just reordering $R_{1}, \ldots, R_{t}$ if necessary) we can assume that $s=1$. By Lemma 21 again, there is $1 \leq s \leq t$ such that $R_{s}$ is a nondividing relation with respect to $\mathcal{M}_{t-1}$ and a dividing relation with respect to $\mathcal{M}_{n}$ for every $n<t-1$. By the previous step we must have $s>1$. Without loss of generality (by just reordering $R_{2}, \ldots, R_{t}$ if necessary) we may assume that $s=2$. If we continue in the same way until $t$ steps are finished we find that each one of $R_{1}, \ldots, R_{t}$ is a dividing relation with respect to $\mathcal{M}_{0}=\mathcal{M}$. Since $\mathcal{M}$ is simple there are (by the existence of nondividing extensions) $c, d \in M$ such that $c \perp \mathcal{M}_{d}$. Then $\operatorname{tp}_{\mathcal{M}}(c, d)$ is isolated by $R_{i}$ for some $1 \leq i \leq t$ and this $R_{i}$ must be a nondividing relation with respect to $\mathcal{M}$, contradicting (via Corollary 19) that every $R_{i}$ is a dividing relation with respect to $\mathcal{M}$. This finishes the proof of Lemma 12 and of Theorem 1

The assumption that $\mathcal{M}$ is binary was used directly in the proofs of Lemmas 17 and 20 and indirectly in the proof of Lemma 21 through the use of Corollary 6.

\section{REFERENCES}

[1] A. Aranda López, Omega-categorical simple theories, Ph.D. thesis, The University of Leeds (2014).

[2] Ove Ahlman and Vera Koponen, On sets with rank one in simple homogeneous structures, Fund. Math. 228 (2015), no. 3, 223-250, DOI 10.4064/fm228-3-2. MR 3294610

[3] M. Bodirsky, M. Pinsker, Reducts of Ramsey structures, in M. Grohe, J. A. Makowsky (eds.), Model Theoretic Methods in Finite Combinatorics, Contemporary Mathematics 558, Americal Mathematical Society (2011).

[4] Enrique Casanovas, Simple theories and hyperimaginaries, Lecture Notes in Logic, vol. 39, Association for Symbolic Logic, Chicago, IL; Cambridge University Press, Cambridge, 2011. MR2814891

[5] Gregory L. Cherlin, The classification of countable homogeneous directed graphs and countable homogeneous n-tournaments, Mem. Amer. Math. Soc. 131 (1998), no. 621, xiv+161, DOI 10.1090/memo/0621. MR.1434988(98k:03080) 
[6] Gregory Cherlin and Ehud Hrushovski, Finite structures with few types, Annals of Mathematics Studies, vol. 152, Princeton University Press, Princeton, NJ, 2003. MR.1961194 (2004c:03037)

[7] G. Cherlin and A. H. Lachlan, Stable finitely homogeneous structures, Trans. Amer. Math. Soc. 296 (1986), no. 2, 815-850, DOI 10.2307/2000390. MR846608 (88f:03023)

[8] Marko Djordjević, The finite submodel property and $\omega$-categorical expansions of pregeometries, Ann. Pure Appl. Logic 139 (2006), no. 1-3, 201-229, DOI 10.1016/j.apal.2005.05.013. MR.2206256 (2006k:03055)

[9] Tristram de Piro and Byunghan Kim, The geometry of 1-based minimal types, Trans. Amer. Math. Soc. 355 (2003), no. 10, 4241-4263, DOI 10.1090/S0002-9947-03-03327-0. MR1990585 (2004d:03078)

[10] Roland Fraïssé, Sur l'extension aux relations de quelques propriétés des ordres (French), Ann. Sci. Ecole Norm. Sup. (3) 71 (1954), 363-388. MR0069239 (16,1006b)

[11] A. Gardiner, Homogeneous graphs, J. Combinatorial Theory Ser. B 20 (1976), no. 1, 94-102. MR.0419293 (54 \#7316)

[12] Ja. Ju. Gol'fand and M. H. Klin, On k-homogeneous graphs (Russian), Algorithmic studies in combinatorics (Russian), "Nauka", Moscow, 1978, pp. 76-85, 186 (errata insert). MR509886 (80d:05043)

[13] Bradd Hart, Byunghan Kim, and Anand Pillay, Coordinatisation and canonical bases in simple theories, J. Symbolic Logic 65 (2000), no. 1, 293-309, DOI 10.2307/2586538. MR.1782121 (2001j:03066)

[14] P. Hell, J. Nešetřil, Colouring, constraint satisfaction, and complexity, Computer Science Review, Vol. 2 (2008) 143-163.

[15] Wilfrid Hodges, Model theory, Encyclopedia of Mathematics and its Applications, vol. 42, Cambridge University Press, Cambridge, 1993. MR 1221741 (94e:03002)

[16] Tristan Jenkinson, J. K. Truss, and Daniel Seidel, Countable homogeneous multipartite graphs, European J. Combin. 33 (2012), no. 1, 82-109, DOI 10.1016/j.ejc.2011.04.004. MR.2854633(2012k:05329)

[17] W. M. Kantor, Martin W. Liebeck, and H. D. Macpherson, $\aleph_{0}$-categorical structures smoothly approximated by finite substructures, Proc. London Math. Soc. (3) 59 (1989), no. 3, 439-463, DOI 10.1112/plms/s3-59.3.439. MR1014866(91e:03033)

[18] Alexei S. Kolesnikov, n-simple theories, Ann. Pure Appl. Logic 131 (2005), no. 1-3, 227-261, DOI 10.1016/j.apal.2004.06.001. MR2097228(2005h:03067)

[19] V. Koponen, Homogeneous 1-based structures and interpretability in random structures, submitted. Online: http://arxiv.org/abs/1403.3757

[20] A. H. Lachlan, Countable homogeneous tournaments, Trans. Amer. Math. Soc. 284 (1984), no. 2, 431-461, DOI 10.2307/1999091. MR743728 (85i:05118)

[21] A. H. Lachlan, On countable stable structures which are homogeneous for a finite relational language, Israel J. Math. 49 (1984), no. 1-3, 69-153, DOI 10.1007/BF02760647. MR788266 (87h:03047a)

[22] Alistair H. Lachlan, Stable finitely homogeneous structures: a survey, Algebraic model theory (Toronto, ON, 1996), NATO Adv. Sci. Inst. Ser. C Math. Phys. Sci., vol. 496, Kluwer Acad. Publ., Dordrecht, 1997, pp. 145-159. MR 1481442

[23] Alistair H. Lachlan and Allyson Tripp, Finite homogeneous 3-graphs, Math. Logic Quart. 41 (1995), no. 3, 287-306, DOI 10.1002/malq.19950410302. MR.1373115 (96k:05144)

[24] Alistair H. Lachlan and Saharon Shelah, Stable structures homogeneous for a finite binary language, Israel J. Math. 49 (1984), no. 1-3, 155-180, DOI 10.1007/BF02760648. MR788267 (87h:03047b)

[25] A. H. Lachlan and Robert E. Woodrow, Countable ultrahomogeneous undirected graphs, Trans. Amer. Math. Soc. 262 (1980), no. 1, 51-94, DOI 10.2307/1999974. MR.583847 (82c:05083)

[26] Dugald Macpherson, Interpreting groups in $\omega$-categorical structures, J. Symbolic Logic 56 (1991), no. 4, 1317-1324, DOI 10.2307/2275477. MR.1136459 (92m:03043)

[27] Dugald Macpherson, A survey of homogeneous structures, Discrete Math. 311 (2011), no. 15, 1599-1634, DOI 10.1016/j.disc.2011.01.024. MR2800979 (2012e:03063)

[28] Jaroslav Nešetřil, Ramsey classes and homogeneous structures, Combin. Probab. Comput. 14 (2005), no. 1-2, 171-189, DOI 10.1017/S0963548304006716. MR2128088(2005m:05225) 
[29] Daniel Palacín, On omega-categorical simple theories, Arch. Math. Logic 51 (2012), no. 7-8, 709-717, DOI 10.1007/s00153-012-0294-7. MR2975425

[30] Anand Pillay, An introduction to stability theory, Oxford Logic Guides, vol. 8, The Clarendon Press, Oxford University Press, New York, 1983. MR719195 (85i:03104)

[31] James H. Schmerl, Countable homogeneous partially ordered sets, Algebra Universalis 9 (1979), no. 3, 317-321, DOI 10.1007/BF02488043. MR544855 (81g:06001)

[32] J. Sheehan, Smoothly embeddable subgraphs, J. London Math. Soc. (2) 9 (1974/75), 212-218. MR.0363974 (51 \#229)

[33] S. Shelah, Classification theory and the number of nonisomorphic models, 2nd ed., Studies in Logic and the Foundations of Mathematics, vol. 92, North-Holland Publishing Co., Amsterdam, 1990. MR.1083551 (91k:03085)

[34] Frank O. Wagner, Simple theories, Mathematics and its Applications, vol. 503, Kluwer Academic Publishers, Dordrecht, 2000. MR,1747713 (2001b:03035)

Department of Mathematics, Uppsala University, Box 480, 75106 Uppsala, Sweden

E-mail address: vera@math.uu.se 